\title{
Sternotomy versus thoracotomy lung transplantation: key tips and contemporary results
}

\author{
Gita N. Mody ${ }^{1}$, Anthony Coppolino ${ }^{2}$, Steve K. Singh ${ }^{3}$, Hari R. Mallidi ${ }^{2}$ \\ ${ }^{1}$ Division of Cardiothoracic Surgery, Department of Surgery, University of North Carolina, Chapel Hill, NC, USA; ${ }^{2}$ Division of Thoracic Surgery, \\ Department of Surgery, Brigham and Women's Hospital, Boston, MA, USA; ${ }^{3}$ Trillium Health Partners, University of Toronto, Toronto, Canada \\ Correspondence to: Gita N. Mody. Department of Cardiothoracic Surgery, Burnett-Womack Building, Suite 3041, Campus Box 7065, University of \\ North Carolina, Chapel Hill, NC 27599-7065, USA. Email: gita_mody@med.unc.edu.
}

\begin{abstract}
The purpose of this report is to provide an updated description of the technique of bilateral sequential lung transplant via median sternotomy. A sternotomy provides the advantage of less morbidity than the clamshell incision, as well as exposure to perform mechanical circulatory support and concurrent cardiac procedures. Our experience shows that lung transplantation via a midline sternotomy can be done with equivalent to better short-term outcomes than a clamshell incision, including earlier extubation and fewer transfusions. Familiarity with this technique is important for all surgeons managing end-stage lung disease.
\end{abstract}

Keywords: Lung transplant; technique; sternotomy

Submitted Jul 02, 2019. Accepted for publication Nov 15, 2019.

doi: 10.21037/acs.2020.01.01

View this article at: http://dx.doi.org/10.21037/acs.2020.01.01

\section{Introduction}

The procedure of lung transplantation has evolved significantly over the last 40 years. The most significant technical change has been from a tracheal anastomosis to a hilar anastomosis in order to avoid bronchial ischemia (1). Details of the operative steps performed vary by surgeon and institution. No matter the approach, an emphasis on early extubation, excellent pulmonary toilet and aggressive ambulation of the patient postoperatively is critical.

Despite the frequent use of both incisions, few studies have compared outcomes after a clamshell thoracotomy (bilateral anterior submammary thoracotomies connected by a transverse sternotomy) versus a median sternotomy for performing bilateral lung transplantation. Better pulmonary function and less pain have been described with sequential bilateral anterolateral thoracotomies compared to the clamshell incision (2). Notably, exposure of the mediastinum may be compromised without division of the sternum in this technique. Median sternotomy for lung transplantation has been well described (3-6) and may allow for a shorter operative time, improved chest wall mechanics and fewer wound complications (7). Additionally, the sternotomy approach frequently requires the use of cardiopulmonary bypass for hemodynamic support, to permit exposure for the hilar anastomosis. Given concerns regarding resuscitative needs post bypass and resulting primary graft dysfunction, a median sternotomy has been avoided in some institutions.

In our experience, routine use of cardiopulmonary bypass allows improved intraoperative hemodynamic stability, decreased warm ischemic time and better controlled cold reperfusion; consequently, the median sternotomy approach to lung transplantation has superior short-term results and equivalent midterm results compared to other incisions. Therefore, we present our operative technique of a sternotomy approach to lung transplant surgery and its results in order to update the literature on this topic.

\section{Operative techniques}

\section{Preparation}

There are a few contraindications to the sternotomy 


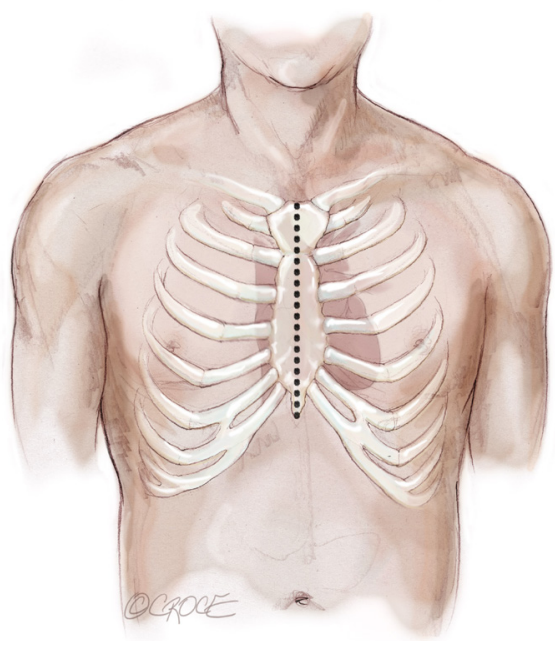

Figure 1 Median sternotomy incision.

approach. These include enlarged hilar lymph nodes from conditions such as sarcoidosis, which complicates the vascular anastomoses. A preoperative CT angiogram should be obtained in these cases to examine the relationship of the calcified lymph nodes to the pulmonary artery (PA). Furthermore, posterior-apical pleural thickening, such as in patients with cystic fibrosis, can be difficult to reach through a median sternotomy. Patients with pulmonary fibrosis and obstructive disease are ideal for the sternotomy approach, as there are few adhesions and the hila are very well visualized through sternotomy.

\section{Exposition}

The recipient is placed in a supine position with their arms tucked. The neck is extended using a shoulder roll. A midline sternotomy is made (Figure 1). The pleura is opened bilaterally. The chest is explored for adhesions, which are freed with electrocautery to ensure hemostasis. The anterior aspect of the hila are prepared by dissecting the superior pulmonary vein and main branch pulmonary arteries, taking care to avoid the phrenic nerve (Figure 2A,B), which is in close proximity to the hilum particularly on the right side. Next, a pericardial well is established, heparin is administered and full cardiopulmonary bypass is initiated with ascending aorta and bicaval cannulation. Superior vena cava cannulation is important to avoid poor upper body drainage during retraction to expose the right hilum. An aortic root vent and PA vent are added (Figure 3). The temperature is allowed to
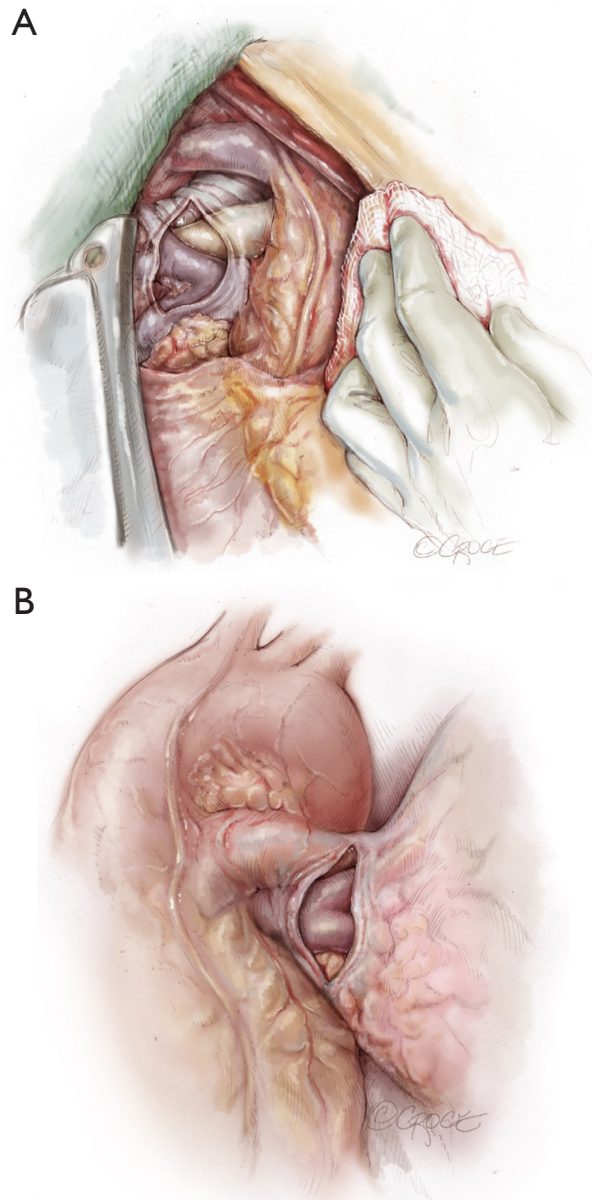

Figure 2 (A) The mediastinal pleura is opened over the right hilum exposing the superior pulmonary vein and truncus arteriosus. The right phrenic nerve is near the hilum and is carefully protected; (B) the superior aspect of the left hilum can be exposed from sternotomy view. However, hemodynamic compromise may limit retraction of the heart to the right until cardiopulmonary bypass has been initiated.

drift to 32 degrees.

\section{Operation}

The hilar dissection proceeds and both pneumonectomies are performed. We staple the vascular structures with an endo-GIA stapler. Attention is paid to hemostasis and controlling lymphatic channels with clips. On the right, the hilar lymph node packet is maintained intact by hugging the bronchus intermedius with the electrocautery. Devascularizing the native bronchus is 


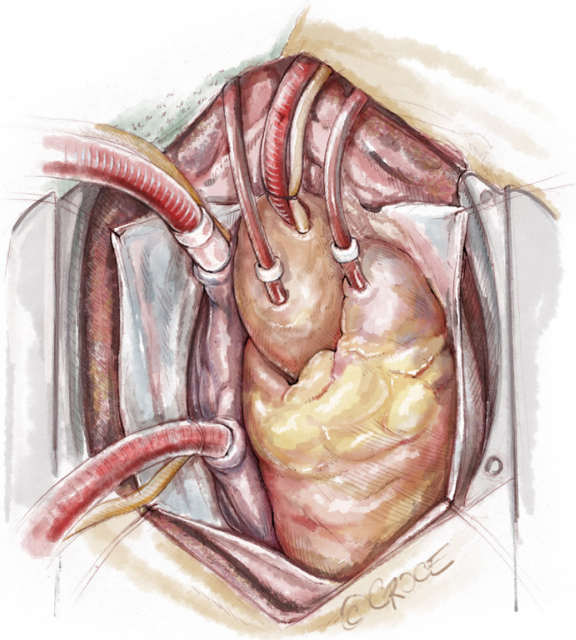

Figure 3 Once ready to initiate cardiopulmonary bypass, the pericardium is opened and cannulae for bicaval drainage, aortic perfusion, and venting the aorta and pulmonary artery are placed.

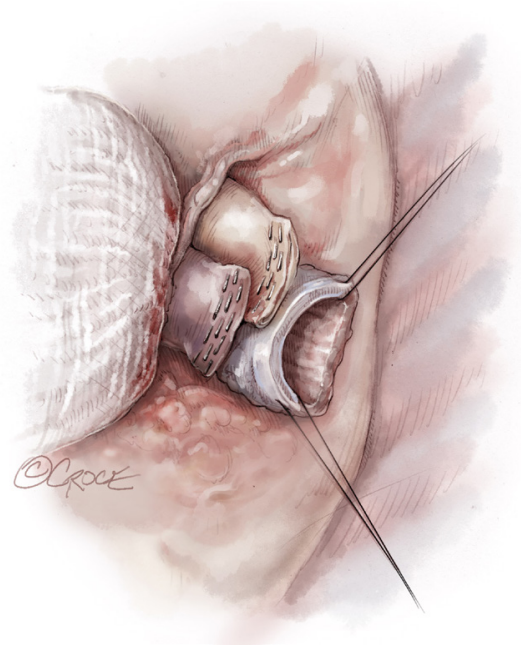

Figure 4 The hilum is prepared by dividing the artery and veins with a stapler and opening, trimming, and placing stay sutures in the bronchus. Prepared left hilum is pictured below.

avoided. The opened bronchus is suctioned clean and silk stay sutures are placed at the junction of the cartilaginous and membranous portions to aid retraction during the anastomosis (Figure 4). The pericardium encircling the PA and left atrium is opened wide in order to lengthen and free these structures.

Once hemostasis of the chest wall has been confirmed and the hila are prepared for implantation, the donor lungs
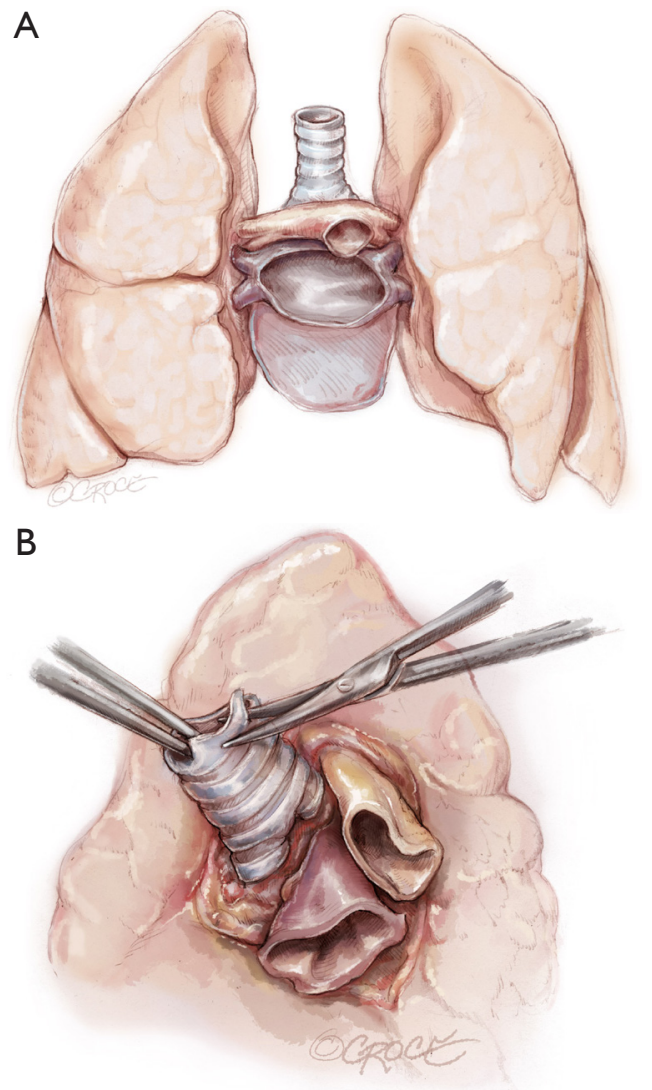

Figure 5 (A) The en bloc donor bilateral lungs are prepared by dividing the pulmonary veins and pulmonary artery bifurcation as well as the posterior pericardium; (B) the mainstem airway on each side is divided two rings above the secondary carina.

are prepared. The left atrial cuff and pulmonary arteries are divided and trimmed. The donor bronchus is divided two rings above the secondary carina in order to avoid ischemia (Figure $5 A, B)$.

The lungs are implanted sequentially, starting with the bronchus using a long continuous running 4-0 Prolene on a SH-1 needle. The anastomosis is done end to end, starting with the posterior membranous portion (Figure 6). The same type of suture is used for the pulmonary vein anastomosis, which is done intrapericardially on the left (Figure 7). Continuous running 5-0 Prolene on a RB-1 needle is used for the PA (Figure 8). On the right, a vascular clamp is applied during the posterior wall of the PA anastomosis, to avoid retraction behind the SVC. The PA vent rate can be modulated to keep the field dry and also allow the first lung to be partially perfused once implanted. The second lung is then implanted in a similar fashion. 


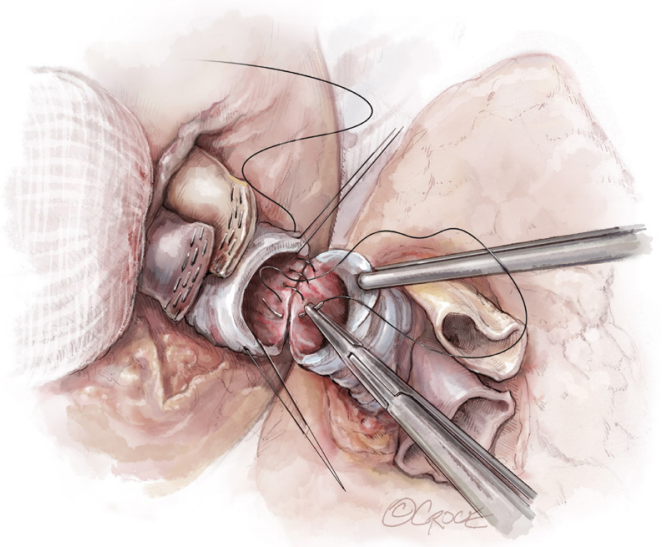

Figure 6 The donor airway is anastomosed to the recipient using running suture starting with the posterior membranous portion.

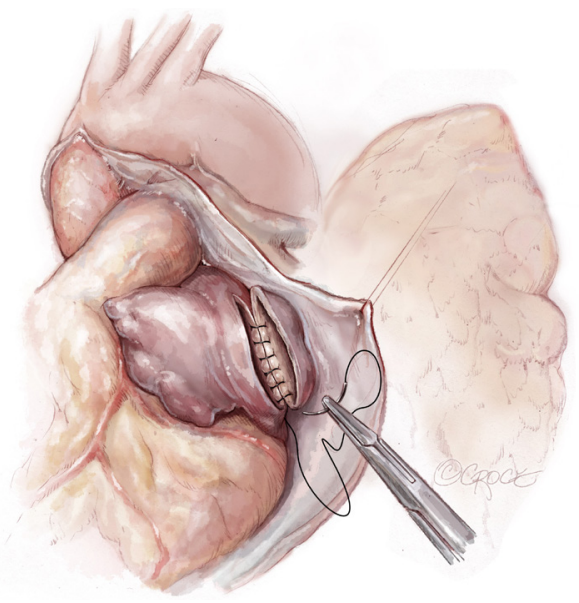

Figure 7 The pulmonary vein anastomosis is done, imbricating the posterior wall of the donor left atrium.

We add a left atrial vent across the second pulmonary vein anastomosis to aid in de-airing.

\section{Completion}

Bronchoscopy is used to examine and clear the airways before ventilating with low tidal volumes and room air. After a period of cold reperfusion and deairing, transesophageal echocardiogram is done to confirm pulmonary vein flow and the patient is warmed and weaned from bypass. Heparin is reversed with protamine. Chest drains are placed in bilateral hemithoraces and the mediastinum. Closure is done with an overlapping figure of eight sternal wires.

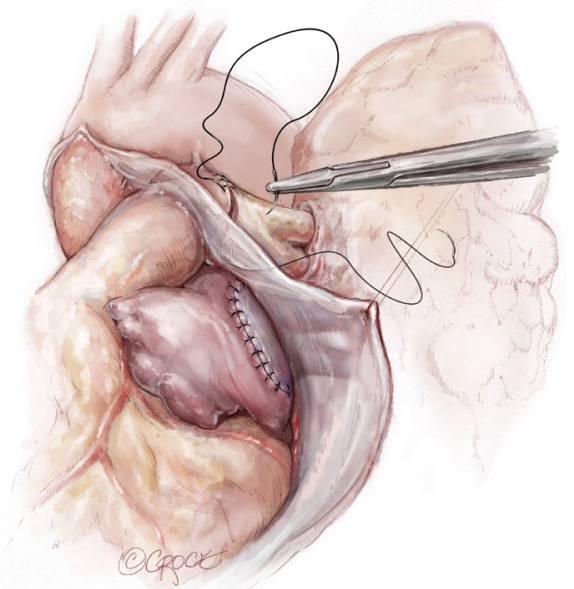

Figure $\mathbf{8}$ The pulmonary artery anastomosis is done in a similar fashion with a running double-armed suture.

\section{Comments}

\section{Clinical results}

We examined short term outcomes of 71 bilateral lung transplant cases done at Brigham and Women's Hospital from October 2013 to January 2017, of which 31 were done by clamshell and 40 by sternotomy. Average case duration (358 vs. 473 minutes) and ischemic time (248 vs. 347 minutes) were significantly shorter in the sternotomy group $(\mathrm{P}<0.001)$. Transfusion requirements were significantly higher for clamshell group with 3.52 vs. 0.85 units PRBC $(\mathrm{P}<0.001)$ and 1.81 vs. 0.65 bags of platelets $(\mathrm{P}=0.05)$. Time to extubation was significantly faster for sternotomy patients with $70 \%$ (28 of 40) extubated by 48 hours postop compared to $39 \%(\mathrm{n}=11 / 28)(\mathrm{P}=0.003)$. Sternotomy maintained statistical significance for association with less transfusions and early extubation when controlling for patient demographics, comorbidities and LAS score. Our study is the largest series comparing clamshell versus sternotomy incisions for bilateral lung transplant and demonstrates improved short-term outcomes after sternotomy incision.

\section{Advantages}

Less soft tissue dissection, better pain control, and earlier mobilization are benefits of sternotomy versus clamshell incision for bilateral lung transplantation. In our anecdotal experience, patients also use less narcotics after sternal incision compared to thoracotomy. Furthermore, recognizing an increasingly elderly end stage lung disease 
population, median sternotomy provides exposure to perform concomitant cardiac procedures as needed at the time of transplant.

With increasing use of marginal donors, sternotomy with cardiopulmonary bypass support also offers the flexibility of central cannulation for postoperative ECMO if needed. We have found that the sternotomy approach maximizes the benefits of excellent exposure of the mediastinum and hilum and full hemodynamic support while minimizing pain and bleeding.

\section{Caveats}

More research is needed to measure pain, quality of life and other patient-reported outcomes after a lung transplant performed by various surgical incisions. Future work comparing prospective measurement of primary graft dysfunction and long-term outcomes will be crucial to informing the use of a particular surgical incision.

\section{Acknowledgments}

None.

\section{Footnote}

Conflicts of Interest: The authors have no conflicts of interest to declare.

Cite this article as: Mody GN, Coppolino A, Singh SK, Mallidi HR. Sternotomy versus thoracotomy lung transplantation: key tips and contemporary results. Ann Cardiothorac Surg 2020;9(1):60-64. doi: 10.21037/ acs.2020.01.01

\section{References}

1. Pasque MK, Cooper JD, Kaiser LR, et al. Improved technique for bilateral lung transplantation: rationale and initial clinical experience. Ann Thorac Surg 1990;49:785-91.

2. Meyers BF, Sundaresan RS, Guthrie T, et al. Bilateral sequential lung transplantation without sternal division eliminates posttransplantation sternal complications. J Thorac Cardiovasc Surg 1999;117:358-64.

3. Dark JH. Median Sternotomy for Lung Transplantation. Operative Techniques in Thoracic and Cardiovascular Surgery 2015;20:87-103.

4. Kohno M, Steinbruchel DA. Median sternotomy for double lung transplantation with cardiopulmonary bypass in seven consecutive patients. Surg Today 2012;42:406-9.

5. Teman NR, Xiao JT, Tribble CG, et al. Median Sternotomy for Lung Transplantation: Techniques and Advantages. Heart Surg Forum 2017;20:E089-E091.

6. Bates $M$, Factor $M$, Parrino PE, et al. Lung Transplantation and the Routine Use of Cardiopulmonary Bypass and Median Sternotomy: Experience at the Ochsner Multi-Organ Transplant Institute. Ochsner J 2017;17:38-41.

7. Macchiarini P, Ladurie FL, Cerrina J, et al. Clamshell or sternotomy for double lung or heart-lung transplantation? Eur J Cardiothorac Surg 1999;15:333-9. 